\title{
EVERARDI VORSTII
}

MEDICINE PROFESSORIS

Oratio funebris

I $\mathrm{N} O \mathrm{O}$ B I $\mathrm{T}$ V $\mathrm{M}$

V. N. ET C L.

CAROLI CLVSII

A T R E B A T I .

Accefferunt

VARIORVM EPICEDIA:

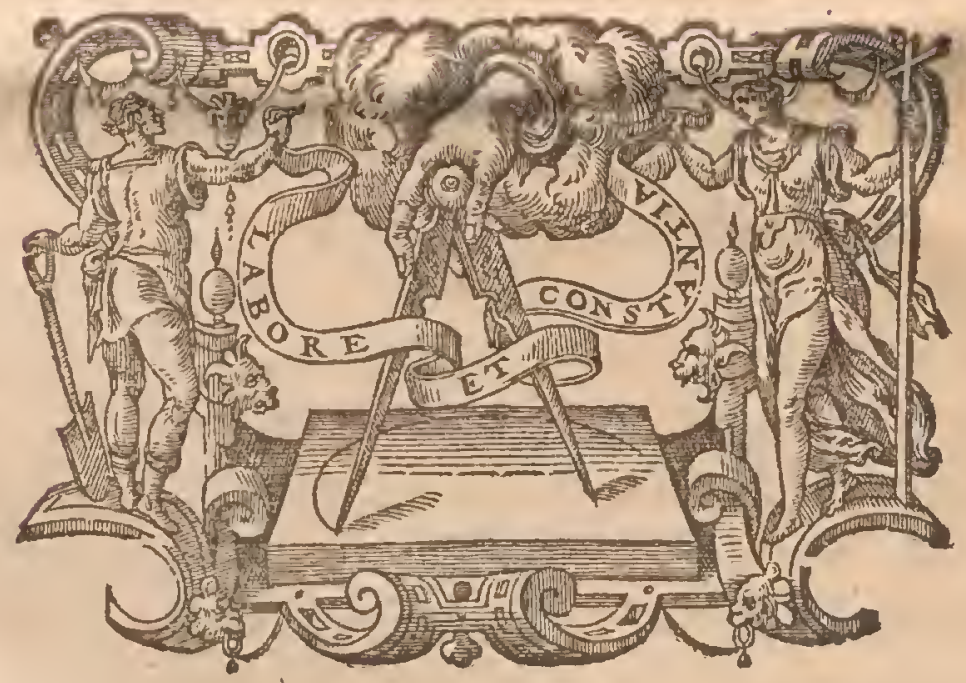

In OFIicina Plantiniana

ReA PHELEXCIF.

$16 \mathrm{Ir}$. 



\section{E VERAR D I VORSTI}

MEDICINE PROFESSORIS;

\& magnifici Acad. Rectoris,

\section{ORATIO FVNEBR IS in obitum}

\section{N. \& CL. \\ CAROLI CLVSII,}

Senatores Academici, Profeffores $\mathrm{Cl}^{\text {mi }}$, Illuftres ac generofi $S$ Barones, Viri Ampliffimi, doctifimi \& reuerendi, Iuuentus ornatiflima atque Reip. fpes florentiffima;

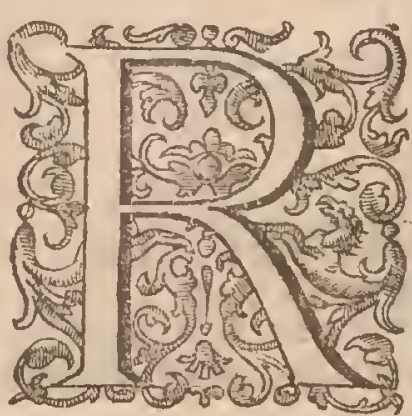

C ENS ac nuperum Academix noftre fatum, alius nunc iterum longè acerbiffinus excepir cafus. Extulimus paucis abhinc diebuș Iofephum. Scaligerum, temporum noftrorum Phoenicém; hunc breti adeò interuallo fub bequutus eft Carolus nofter Clufius; vixdum dolor nófter aliquantò fubfederat, ecce noù percellimur luctu, malúmque paullò remiffum rurfus integrafcit. nec enim vno contenta Scaligero (in cuius tamen iacturâ rorus ingeniuji orbis) mox \& alterum: hoc nobis fubduxit ornamentum inuida Parca. Huic venerando feni hodiè pro veftrâ pietate parentaftis; huius honorandre memorix huc frequentes conueniftis; huius defuncti gratiâ foris fordidatos, intus mærore obfeffos vos maftus intueor; huius denique caursâ in hunc locum, non tam meâ voluntate, quàm veftrî, Patres Academici, petitione atque impulfu afcendi. Quàm durum fulceperim onus,non me fugit. dieturus enim frum de eo Viro, cuius virtutes, cruditio, in remp. litterariam merita,omnem meam dicendi vim, omnem fupelleCilem excedunt longè : dicturus fum in eo loco, in quem nibiladferri folet, nibil debet nifi perfectum ingenio, claboratum induftriâ, $\&$ quod demorfos fapiar vngues. à quo dicendi fplendore tamerfi quàm. longiffimè hanc meam Orationem abeffe probè fciam, veftrâ nihilominus fretus bencuolentiâ multus in me excufando a pud vos non ero. excufabit me Senatus Academici decretum, quod dicendi hanc impofuit neceffitatem.excufabir pietas, quâd defuneti manes profequutus, extremú hoc officium à medefideraripaffus non fuilexcuifabit Viræac 
piofeffionis mex genus quod ab amoenioribus iftis litteris \& eloquentix ftudïs multum femotum eft. excufabit demum temporis anguftia, quæ meditationi accurationi fpatium perexiguum conceflit. Quare fi non ex dignitate omnia dixero, \& $\mathrm{cx}$ tam multis pauca folù detbauero, haud fpero apud Auditores tàm æquos iudicia mihi vlla fore parata iniquar. Fauete igitur piis conatibus, Auditores humanilimi; fauorisque veftriauram tantifper mihi concedite, dum que do tanto viro $a b$ aliis aliquando ornatiùs dici aut feliciùs fribi poterune,

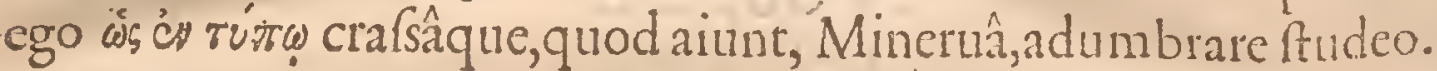

Qui viros preclaros laudandos fibi fufcipiunt, à folo natali in quo orti, in quo primùm haufcrunt calum, orationes fuas au fpicari folent non immeritò; huic enim tantum à maioribus eft tributum, ve parentis fanctiffimum nomen nobifcum commune habere voluetint, nibilque prorfus effe, quod à nobis vel fortiter geri, vel prudenter agi, vel fapienter dici poflit, cuius patria fibi non arroget iurc fuo partem, zquiflumi rerum xftimatores cenfucrunt. Atrebacum vrbs Gallia Belgicx ad Scarpum fuuium antiquifima eft, ampla, elegans, amocna, aëre faluberrimo, ingeniis ac nobilitate florens; torius gentis $M c-$ tropolis iam olim habita in hac Clufus nofter nafcitur A tino falutis millefino quingentefimo \& vigefimo fexto, menfe Februario, patre Michaële dé Efclufe, Domino de Warenes, \& Confiliario Curix Prouincialis: matre Guliclma Quineaut, rari exempli foeninâ. quo in lóco parenteś fimul cum animâ illi tradiderunt, patriam non obfuram, familiam won poenitendam, dotrum innocentem, opes honeftas, ingenium liberale, ftudisqúu oprimarum artium capeffondis aptiflimum. Quare vt primum ex ephobis exceflit, Gandauum à parentibusmiffus, frib optimis preceptoribus iis artibus, quibus xtas illa ad humanitatem informari folet, ritè im. butus elt. quo quidem tempore multa nequie ca vulgaria fpectatx indolis edidi fpecimina. Erate iam grandior factus, ad grauiores difciplinas \& imprimis Ius Ciuile dif́cerdum, Louanium totius Europx florentifimum tunc Gymnafium, ac litterarum Emporium ablegatur : vbi celebriores in omn: fcientiarum genere Viros publicè docentes cupidiffmè audiuir; priuatim etiam quibufdam ex precipuis \& familiariter vfus, alfiduâ illa doctorum hominum confuetudine effecit, vt breui admodum infi. gnem optimarum rerum peritiam, nomenque inter xquales clarum fibi compararet. Erat enim eâ tunc in vrbe fummus præfantiflimo. rum virorum \& adolefentum concurfus ex omnibus Europx prouinciis; \& docentium difcentiumq́ue fudia ad æmulationcm æquc feruebant. Anno xtatis vigefimo fecindo Louanio digreffus, ad v beriorem ingenii cultum capiendum, \& externarum regionum vifendarum cupidine Alagrans, in Gcrmaniam contendit, \& Marpurgi 
apud Chatros aliquandiu fubticic, vbi Oldendorpium clariffiminu apud fuos \& exteros I urif́nónfultum, diligentiffumè audiuit: ibidémq . in familiaricatem incidit Andrex Hyperii, magnx exiftimationis ac famx apud Germanos Theologi. Huius confilio anno infequente Wirtebergam Saxonú adiit, potiffimùm ve Philippum Melanchthonem videret, audiret \& corain cognofceret; virum mehercle egregium, qui f́cientiarú orbem vniuerfum vel folus, vel cum paucis apud populares fuos intellectione cóplexus eft; \& ob id, dum viucret, Germanix fuæ Oraculum habitus. Circa annum Chrifti cIo. Io. s. Wirtebergâa profectus Francofurtum indeque Argentinam petiit, vbi paullulum. commoratus, cùm Gallix luftrandx teneretur defiderio, per Heluetios \& Allobroges Lugdunum peruenit, hinc Montepeffulum: in qua vibe certo confilio triennium totum egit, Gulielmi Rondeletii Medici celebratifimi contubernio ac mensâ vfus. Hoc primùm in loco illum cepir Medicina tirpiumq́ue nofcendarum amor, quoad extremum vfque vitx diem raptus, Iuris Ciuilis profeffioni nuncium remifit, feque totum Sacræ Artis, \& prxcipuć fumplicium medicamentorum cognitioni tradidit, infito quodam naturæ ductit affiduus ac fedulus Rondelctii, cui vnicè carus \& acceptus erac, auditor, ex ore docentis excepic Methodum curandorum morborum particulariums quam poftea ty pis vulgauit Laur. loubertus. Reiherbarix ftudio fu-s pra modum delectarus, frepiffimè in agrum \& circumuicina loca prodibat, totamque Galliam Narbonenfem perreprabat: neque, quod multi peregrimantes hodie faciunt, perfunctoriè aut veluti canis $\mathrm{Ni}$ lum lambit, exteras regiones obibar, verùm penfrculatè minurifima etiam quæque animaduertebar, nec folùn rerum naturalium ibi nafcentium indagarione contentus, quicquid prarerea ad fitum, antiquitates \& populorum mores fpectaret, mente \& oculis attentifiunis - obferuabat. cui inter catera fidem facit Tabula illius Chorographica Gallix Narbonenfis, quam Theatro fuo Gcographico Abrahamus Ortelius inferiiit. Dum apud Montepeffulanos degerer, Petrum Lotichium Poëtarum Germanix principem cognouit, \& inter reliquos amicos familiariffumè coluit. huius ad Clufum verfus aliquot \&E Epiftolx cun reliquis cius operibus leguntur. Porro in fudio Botanico cuius incredibili Hagrabat amore, ita breui profecit, vt vel tunc Rondeletio, Scyionio aliisque Academix illius Doctoribus liquerer, quantus in illa profeffrone aliquando futurus effet : non obfcuris quippe. indiciis colligebant, ad rem herbariam ornandam, augendamque natum atque vnice factum. vtpotecuius animo altillime infixum hærebat graue illud diotum quod apud Galenum extare nouimus; \& a ab Oribafio repetitum legimus, fimplicium medicamentorum, \& facultatum qux eis infunt cognitionem adeò Medico neceflariam effe, vt

a 3 fine 
fine eâ nemo ritè mederi queat. Equidem ita iudico; fi Medici alioquin eximii animum herbarum notitiâ fpoliaueris, mentemáque natiuâ ftitpium fpecie nudancris, licet cælo deiêtus, licet Efculapius ipfe fuerit, licet omni Mufarum choro ftipatus, Phyfica, Anatomica atque Chirurgica callens, placere tamen poffe ne Apollini quidem fuo,vtpote herbarum feientiflumo. Fuit fanc̀ hre Sapientix pars quæ firpium hiftoriam complectitur, non ita multis ab hinc annis in obfcuro planè pofita,denfinfimisquue inuoluta tenebris. nam præterquam quòd per fe fat difficilis effet, temporum longinquitas \& crebrx populorum migrationes, in caufsa fuerunt, vt pleræque, ne dicam omnes, plantæx veteribus abiectis noua fxpiùs induerint ac comnutârint nomina, ita ve priftinæ nomenclaturæ nec vola nec veftigium appareat vllum. Hinc etiam morofior ac fcrupea prorfus reddita, quod prifci materiæ medicx Scriptores, ftirpium, quas pro vulgò cognitis habebànt, paucas inter defcribendum, non rarò nullas tradiderine notas, quibus à pofteris recognofci poffent. Quibus \& tertium acceffit incommodum, quòd recentiorum quidam ad primam quamque vel leuifimam notam à veteribus appofitam, alicnas ac nonnunquàm contrarias fupponunt plantas, adeò vt loco falutarium remediorum, venena eriam quidam nobis furrogârint nocentiffima, damnabili prorfus atque fceleratâ infcitiâ. Quibus malis ne diutiùs conflictaretư̆ Ars medendi, fummi Numinis prouidentiâ factum, vt patrum. noftrâque memoriâ multi preclari exorti fint Viri, qui hanc Philolophix partem illufrandam fibi fumpferunt; inter quos primas obtinent Leonicenus, Ruellius, Matthiolus, Fuchfius, Tragús, Dodonæus, Dalechampius, Lobelius, Cafpar Bauhinus, Clufus denique hic nofter; de cuius in hoc ftudiorum genere excellentiâ. \& fingulari peritiâ non eft quod multis apud vos differam; res ipfa loquitur, \&r qua omnium manibus teruntur, Viri eminentiflimi Scripta id a bundè teftantur. inde enim apparet duo fibi potiffimùm propofita habuiffe, vt ab antiquis defcripta aut memorata, altâqueiam caligine merfa, erueret atque in lucem protraheret; noua verò aliisque indicta ipfe reperire poffet, repertaque optimâ fide bono publico omnibus communicare. vtrumque feliciffimè percgit, \& cum immortali nominis fui gloria. perfecit. Verùm ad reliquum de Vitâ Optimi noltriSenis fermonem regredior. Montepeffulo, poft gradum, vt vocant, Licentia in Medicinâ adeptum, difédens per Galliam Lugdunenfem, Geneuamq́ue, iterùm Bafileam peruênit, vbiexquifitiffimis honoribus ab Ordine Academico exceptus, fecundo $R$ heno vectus Coloniam víque Agrippinx, inde terreftri itincre Antuerpiam petiit, \& 2 a b anno quinquagefimoquinto fupra millefimum quingentefinum vfque ad annum fexagefimum terrium in Belgio ferè hæfit, nifi quòd Lutetiam Parifio- 


\section{in OBItVM Caroli Clvsió}

rum fernel excurrere illi vifum. Vertic intereà temporis in feímònentus Gallicum Vicas Hannibalis \& Scipionis à Donato Acciaiuolo Latinè priùs editas, qux inter opera Plutarchi Gallicè euulgata hodiè leguntur. Dodonxi prætereà Plantarum hiftorian in candem linguam. conuertic. Anno verò fexagefimotertio Auguftam Vindelicorum pe-: tiit, proximoque repetiit, \& inde in comitatu 11 lutrium fratrum Fuggerorum,per Belgicam, ceteramque Galliam Oceano proximam,in. Hípanian profectus elt; quam vniuerfam peragrauir \& accuratiflimè perlußtrauir à Pyrenxis montibus ad fretum vfque Gadiranum : quo in itinere ad Calpen equo dilapfus os ribia confregit Olyfipone cùm effet,incidit fortè fortunâ in Dialogos Garçix ab Orta Lufitanicâ linguâ confcriptos, Aromatum apud Indos nafcentium defcriptionem. complectentes, quos cùm auidè percurnffet, dignosáise qui toti orbi innotefrerent iudicaffer, Latinè vertit, \& parergis omiffis contraxit, additis ctiam Notis. Hos primùm anno feptuagefimoquarto ChriftophoriPlantinitypis Antuerpix publicauit: quibus adiuix it Nicolai Monardis Medici Hifpalenfis de Simplicibus med. Noui Orbis Librum ex linguâ icidem Hifpanicâ in Latinam traductum. Hifpaniâ totâ decursâ in Belgium reuerfus elt, $\&$ in eo viquc ad annum feptua gefimum commoratus eft, amicitiâ multorum preftantium Virorum. vfus, Boyfotti, Brancionis, Laurinorum fratrum, Plantini, Raphelengii, \& aliorum. Hoc tempore ex fermone Italico in Latinum tranfrulic Antidorarium, ve vocant, Florentinum; quod anno Lxvil: apud Planrinum édi curauit. Anno verò Lxxı. in Britanniam nauigauit, indéque redux biennio exacto ab Imper Maximiliano I1. A ugufto Viennam Pannonia houefifrm is conditionibus euocatus fuit, \& numero familiarium aulicorum adıcripeus, commisâ illi etiam. Horti Cafarei cura: in quo munere ad annos circirer quatuordecim durauic, in fummâ Maximiliani Aug. \& Rudolphi-F. exiftimatione ac gratiâ.quo tempore Pragam vna cum Imp.Maximiliano profectus eft; quo ibidem virâ defuncto, à Rudolpho fucceffore priftinum illi munus confirmarum fuit. Hungariam ctiam, totamque Aufriam peragratic. Britanniam etiam permiffu Imp.iterum adic: vbi in familiaritatem incidic generofffimi Viri Philippi Sidnxi \& Francilci Drake; à quo fociisq́ue eius naualibus permulta didicit qua ad Exoticorum hiftoriam fpectant. In aulâ Cáareâ quamdiufuic, torum inueftigationifirpium fe dabat, eiusquue rei causâ fapiffimè in. vicinos Pannonix agros montesque excurrebat. Hyberno verò rempore, \& quoties otium illi effer affiduè aliquid quod ad fudium hoc pertineret commentabatur. Edidir enim Anno rxxyr. typis Plantinianis Hifpanicarum firpium à fe obferuatarum Hiftoriam; prater Garçize ab Orta Dialogos, Chriftophori à Colta Hiftoriam Aroma: 
tum, \& Nicolai Monardis Noui Orbis Simpl.med. Anno autem 83. Plantarum Pannonicarum obferuationes. Verfabantur eodem tempore in aulâ Io. Sambucus, Iul. Alexandrinus, Crato, Dodonæus, aliíque non pauci Viri doctiffimi; quos omnes amore fibi deuinctos habuitr. Patuit etiam illi adicus in multorum principum \& illuftrium Virorum amicitiam; inter quos potiffimùm colebat, maximeque familiares habebat Comitem à Serotin, \& Baronem à Batthyan, Hungarix proceres. Aulicx tandem vitæ pertæfus, Anno Ixxxvir Francofurtum ad Mænum conceffit; ibique per annos fermè fex priuatim. egit, fibique vixit: nifi quòd interdum ad Principem Gulielmum Lantgrauium commeare foleret: cui tantoperè acceptus fuit, vt annuo illum honoraret ftipendio; tantoperc̀ familiaris, vt eấdem cùm illo veheretur rhedâ, intimeque confuef́ceret. complures etiam alios in hoc feceffu, vt crat leniffim confuetudinis homo, comparauit fibi amicos, magni nominis regum ac principum Legatos. Illîc cùm effet, vertic è lingua Gallica in Latinam P. Bellonil Obfernationes, quas Anno Lxxxix. publicauit, addito eiufdem Bellonii Libello de neglectâ ftirpium culturâ. Demùn Anno xcur. ab Academix noftrx Curatoribus honorificis litteris \& ftipendio invitatus, huc venit. Ita enim iudicabant Viri prudentiffinit, à tam clari hominis præfentiâ, non minimum fplendoris Academix huic acceffurum: nec opinione fua frufratifunt: quamdiu enimapud nos fuit quá publicè quá priuatim vtilis effe nullo vinquam tempore deftitit. Scripta fua omnia ad incudem hîc reuocauir, extremamq́ue manum addidit, \& in duos tomos digeffit, titulis Hiftorix Rariorum Plantarum, \&c Exoticorum:additis etiam aliis quiburdá libellis, E piftolis \& obferuationibus. Nec folùm fcriptis xternâ memoriâ dignis illuftre reddidit Gymnafium hoc: fed quotidianis fermonibus omnes qui ad ipfum ventitabát erudiebat, benignéq. omnia fua commonitrabat atque communicabat.adéo vt cui conferre cùm illo libéret, nón inter plantas verfäri, fed in Philofophire finu conquiefcere fibi videretur. Erant quippe in hoc viro prater ftirpium cognitionem exactifimam, conplures alix $\&$ infignes dotes. Linguarum prater Griecam \& Latinain, Hifpanicx, Italicæ \& Germanicæ callentiflimus fuit: \& qui Italiam nunquam viderat, optimè tamien illius gentis loquebatar \& f cribebat linguâ. Hiftoriarum \& Geographix peritiflimus. memorix tàm felicis, vt quxcunque inter peregrinandum etiain minutiflima quixque obferuaffer, ad vinguem. teneret $\&$ tanquam in numerato haberet. Pietatis in Deuín atque hómines non obfcura religuit indicia. ipfe cùm màior natu effet, fratri tamen minoriceffit vltrờ feudo ac dominio Watenefri ; exemplo noftris hiife moribus raro. Attritis pratereà fuorum ob bellum noftrum ciuileopibus, liberali manu fubuenit ex co quod frugali fuấ \& calibe virâs 
virâ reliquñu frbifecerat: cibi enim potusúuc defiderio nàturali,non voluptate, modus illi finitus erat; ab corum ingenio procul, quorum, corpora in faginâ, animi in macie \& veterno obtorpefcunt. Tranquillitas porto animi ac ferenitas in illo incredibilis, quam longovfu fibi pepererat ex bonâconfcientiâ, rectis atque honettis actionibus, contemper fortuitorum, placido vitæ \& continuo tenore, vuam candemáue perpetuò premente viam. Temporis tàm diligens difpenrator, ve nunquam deprehenfus fit, extremâ criam illâ xtate, quin aut le. geret aliquid aut commentaretur. Amicorum cultor religiofifimus; quos illi plurimos omnis ordin is conciliauit, prater doctrinam, multa cius atque rara virtus, animique manfuetudo. ita vt vix dies præteriret quo non aut ipfe litteras daret, auc ab aliis acciperet, etiam ex remotiffimis regnis ac prouinciis. Summis Principibus quanto in pretio quamq́. carus fuerit, vel ex folis litteris eorum ad ipfum foriptis abundè colligere eft. ita vt yix credibile fic hominen priuatum cxterumá: in tanta maximorum Virorum dignarione vixiffe. Toftremis annis duos ex amicis præcipuè obferuauit, lofephum Scaligerum \& Vincenrium Pinellum, quorum exceffu ita bonus indoluit Senex, yc deinceps manifeftè in peius labi \& à priftino vigore fenfim remittere vifus fir. Valetudo illiab ineunce xtate varia,nec fatis profpera. Francofurti in luxationem coxendicum incidit: quod incommodum eo folùn nomine molẹtuùs ferebat, quòd non, y antè, montes camposque inuife re; plantasque indagate liceret. Herniofus etiam poltremó factus eft: fenfibus alioquin integ in extremo illo fenio citra ocularium adiumentum oprime adhuc le geret, cala maque pingeret. Tanựn tongâ arate \& annorum decurfu feflus,poltquàn iam expleffer annum octuagefimum quartum, ex: tremum claufit Clufusdiem. Vir, abfit inuidia dictis, paucis conferendus, vitât ianocentiflimus, ingenio florentiflimus, propofito fanctif-

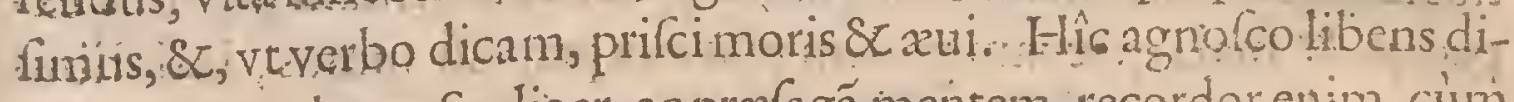
uinam tuam, beate Scaliger, ac prafagá mentem. recordor enim, cùn paullò ante obitum tuum Clufus per internuntiú fefe excufarer quòd valetudine impeditus inuifere te agrotantem non poffer, $1 \mathrm{refpondiffe}$ te; Viueret, valeret tantifper; breui fe vifuros iterum in beatorum fede. Verus, a himiun verus vates: quantulo enimtempore Clufum precellit ingens illa anima? vix altero elapfo menfe fidiffimum fuum fequitur affequiturque Scaligerum Clufus. Quàm forti verò ac conftanti animo fatum fuum excepit? quàm placidè fupremum illud momentum \& quàm auidè captauic ? Quidni verò captarer homo in Chrifti Seruatoris fcholâ enutritus, $\& \tau$ in fpem beatærefurrectionis erectus? meminerat probè, animóque fuo proculdubio tunc voluebar, dietumillud Iobi, confolation is pleniffimum: Scio quòd Redemptor 
meus viuit, \& nouiffimo die de terrâ furrecturus fim; \& rurfus circurndabor pelle meâ, $\&$ in carne meâ videbo Deum. repofita eft hæc fpes mea in finu meo.

Talem tantumq́que Virum amifmus, Auditores; cuius fanè beatirudini vti nos inuidere impium planè foret, ita rurfus noftrum nos lugere cafum nulla vetat religio. Te quidem, ô beate Clufi, ô fortunate Senex, non eft quod lugeamus, qui fempiternâ apud fuperos iam frueris gloriâ: \&r apud nos in fummâ nominis celebritare vixifti, viuesque etiam poft fara æeternùm: eos verò atrigifti annos quos noftrûm aliquis vix f́perare auderet. Et quid fi non atrigiffes? noras mortalium conditionem, cui nafci contigeric, mori reftare; interuallis nos diftingui, exituxquari: vtque mori neminem contingit qui non vixerit; ita nec vivere aliquem poffe, qui non fir moriturus: At verò noltram nos dolemus iacturam, noftrum totiusq́ire reip. litteraria irrecuperabile damnum. Luget Academia Cratenâ, Theophrafto, Diofcoride fuo orbata. Luget ftudiofa Iunentus, \& A Aclepiadum foboles tanto rei Botanicx reftauratore ac patre deftituta. Quin neque omnis intra loci huius fepra confifir dolor, claudique fe patitur tàm angufto fpatio; cmanabic hinc in vniuerfam Europam, imò ad extremos Indos vfque diffundet felongè lateque trifie hoç nuntium. Lugebit ipla parens resum Natura Myften fum Clufum, cuius ingenio atque induftria, opes fuas reclufas 2 tam accuritè defcriptas paullò antè lretabatur. Quare cxceflifti tu quidem à nobis, beate Senex, \&x ad authorem originis ture latus reucrfus es, fed nobis omnibus defiderium tui reliquifi triftifimum, fimulq́ue memoriam nominis tui nunquam interituram.tua enim te monimenta, tua te virtus, ac ferix aliorum laudes, non leuis hæc mea ac breuis predicatio, perpetiò celebrabunt. Deum Sofpitatorem fupplicibus votis oro, obfecro, obteftor, vti. volens propirius fofpirer A cademiam hanc, tantisque circumuentam luctibus expediat, \& mala qux iám pridem plurima perpeffi fumus, latioribus aliquando compenfet euentibus. Ita voueo, ita auguror. Dixi.

\section{Habita fuit bac Oratio in eAcädemiâ post ductum.} funus, die VII. April. 'Anrio CIJ. I0: 'Clx. 
Epitaphivm Clariß. Viri Caroli Clusil, qui Sepultus est Leida in templo Beata Maria.

BONE MEMORIE CAROLI CLVSI I A T REBATIS

\section{P. OS.}

QVI OB NOMINIS CELEBRITATEM PROBITATE, ERVDITIONE, TVM REI INPRIMIS HERBARIÆ I LLVSTRATIONE PARTAM, INTER AVL\& CIES. FAMILIARES ALLECTVS; ET POST VARIAS PEREGRINATIONES A NOBB. DEMVM ET AMPLISS.DD. CVRR. ET COSS.IN HANC VRBEM CONDECORAND E ACADEMIE EVOCATVS,ET STIPENDIO PVB. PER ANNOS XVI. HONORATVS, XXCIV“ E. S. ANNVM INGRESSVS OBIIT CELEBS IV. APR. M.DC.IX. 
A N N O à nato Chrifto M. D. \xy I menfis Februarii XIx fub horam v. ante meridiem natus eft Carolvs Civsrys Atrebatimetropoli Comitarus Artefix in Gallia Belgica. ifticạue in pucritiâ educatus : Gandauum indc-ablegatus, vt operam politioribus literis daret, fub Paulo Euchario viro doctifimo, apud quem bienno vixit:anno xLvı Louanium profeaus Latinx \& Grac $x$ Lingux profeffores in collegio trilingui fiue Bunlidiano, audiuit. Deinde (fic volente patre) Itri Ciuili operam dedit fub C.V. Doctore Gabriele Mudxo:fcd cum animus effer peregrinas regiones luftrandi, Germaniam triennio pòft adit: vbi ex magni \& celeberrimi viri confilio \& autoritate ftudii rationem mutans, neglecta lurifprudentia philofophicum ftudium amplexus cff. Anno quinquagefimo in Narbonenfem Galliam fe contulis : vnde paucos poft annos, ob bellum inter Carolum V. Cæfarem \& Henricum II. Gallix regem exortum, in patriam à patre reuocatus, illîc aliquot annos vixit, donec anno $\mathrm{LX}$ denuò in Galliam reuertere. tur, Lutctiæque biennio viưcret. Inde propter inte?tinum bellum, Aureliâ à principe Condæo occupatâ, Louanium abiit, \& iftîc anno vixit. Anno is 64 cuocatus in Germaniam, bimcftri iftic tranfacto, per celeres equos in Belgium rediit, \& inde per Galliam in Hifpaniam profectus cft, cuius plures prouincias atque ctiam magnam Lufitanix partem annuo fpatio perluftrauit. Anno fequentein patriam reuerfus vfque ad $7 \mathbf{I}$ in câ permanfit, quơ Lutetiam profectus, \& inde per Caletes in Angliam. Illâ autem ex parte peragratâ, denuò Lutctiam \& in Belgium regreflus cft : iftic hxrens vfquc ad annum 73 fupra millefimuñ \&z quingentefimum. tum enim à Cxfare Maxæmilinno huiius nominis II. euocarus Vicnnam Aufrix profectus eft, \& iftic in aulæ Cæfareæ familiarium numerum benignè receptus fuit. quo etian loco apud Cxfarem Rudolphum 1. Maxxmiliani filium fuit. Vixit porrò Vicnnx vfque ad annum $I X X X V I I$ I. (fufceptis tamen intereabins in Angliam peregrinationibus) quo Francofurtum ad Monum fe contulit: $x c ı i$ autem anno Lugdunum apud Batauos $a b$ ordinibus confæderatarum prouinciarum euocatus, eò peruenit annum xtatis agens 68 ; vbi adhuc viuit cclebs annum agens 7 \}. In pueritiâ $\&$ adolefcentiâ variis merbis obnoxius fuit, prxfertim febribus continuis aliquot annis, omnibusçue diuexatus eft, prxterquam quartanâ \& peftilente. Annuna agens 24 hydrope laborauit ex intemperante aqux frigidx potu; quo morbo Cl. V.. Guil. Rondeletii med. celeberrimi \& profefforis in academia Montepefulana, curâ dilıgentiaque liberatus fuic. A nno aurem athtis xxxix in Hifpanca peregrinatione, rucnte in curfu per accliue equo cum ipfo feffore; dextrum brachium fupta cubitum fregit; \& eum infequente, dextrum crus. Viennx porrò A uftrix, annum agenti 55 : pes finifer illiluxatus, \& mallcolus fractus, Octennio deinde pòft Francofurti ad Mœnum, ex cnfu dextrum femur luxatun, \& luxatione nec à Medicis nec à Chirurgis agnita fedneglecta, ad contufionem cumtaxat medicamenta applicantibus, prorfus claudus effectus eft, vt ne pedem quidem promouerc poffit, nifi gemino fcipione fub alis fubnixus. eam ob caufam cùm corpus exercere nequeat, fed perpetuò illi fedendum aut decumbendum fit, graues alii acceffere morbi;calculus, coli dolor, \& hernia. Italicam profectionem ter tentauit:Florentifim am enim illam regionem videre fummopere auebat: fed irriti femper fucre conatus: prim ùm Montepeffulano Mafiliam profectus eft: deinde ex ea vrbe rediens, apud Allobroges hrfit fere femeftre, parentis refponfum exfpectans:peftremò Auguftam vfque Vindelicorum profectus; vnde curfum conuertere debuit in Hifpaniam. Quxadeò caufa fuit, vt adeundi Italiam omnem cogitationem in pofterum deponerct, exiftimans id in. ftitutum Deo non probari, cuius voluntati obfiftere nephas duceret. Eum ob tor animi \& ingenii virtutes atque dotes incomparabilcs, Imp. Maxxmilianus II. Nobilitatis priuilegiis optimo eius merito cohoneftatum, plurimum amabat. Linguarum v I I, vtpote Latinx, Grxcx, Italic $x$, Gallicx, Hifpanicx, Lufitanicx, \& Belgicæ, exactam habctperitiam: variarum autem rerum proftantiflimarum, pracipuè verò in hiftotia fimplicium peregrinorum tantam notitiam \& vfum fibi acquifiuit, vt per tot regna orbis tcrarum fcriptorem earum vel grauiorem vel diligentiorem hodie non reperias. Hiftoriartimvulgd in. cognitarum \& feriptor \& cenfor acutiffimus, vit \& Cofmographix doctiffimus. Scripfic $\&$ vertit hiftorias Americanas ex Hifpanico Idiomare, in Latinum fermonem. Dialogos fiue hiftoriam Garcix ab Horto, proregis Indix medici, Lufitanicâ linguá fcriptam de aromatis \& fimplicibus aliquot medicamentis apud Indos nafcentibus, Latino fermone in epitomen contraxit, \& iconibus ad viuum exprefrs, locupletioribusćue annotationibus illuntrauit, 
illuftrauit, qự quarta edicione apud Plantinividuam catigatior \& auctior produic in 8. anno 93. Librum Chriftophori à Colta medici \& chirurgi de Aromatibus \& medicàmentis in Orieñtali India nafcentibus ex Hifpanico fermone Latinum fecit, \& in epito men contractum quiburdam notis illuftrauit, qui altera editione apud Plantinividuam Antuerpix anno 1593. in formá octaua publicatus elf auctior \& caftigatior. Hiftoriam Nicolai Monardi Hifpalenfis Medici duobus libris. feriptam Hifpanico fermone de fimplicibus Medicamentis ex nouo orbe delatis, quorun in medicina vfus eft, Latio donauit, \& in vnum volumen contractam, annotatione \& Iconibus affabrè depictis, illuftrauir; qua tertia editione excufa eft ibidem ex poftrema authoris recognitione formâ octauâ anno 1593. Idem fecit tabulam Hifpanix, antiquis \& recentibus in ea locorum nominibus infetiptis; quam-fe editurum promifit Ortelius. Eiufdem Hiftoria aliquor ftirpium per Hifpaniain obferuatarum libris duobus expreffa eft Antierpix in 8: Anno 1576.

Hec Boiffardus: quibus vitam Clarißs. Clufil vfgive adannum is 93 compendio defcri-

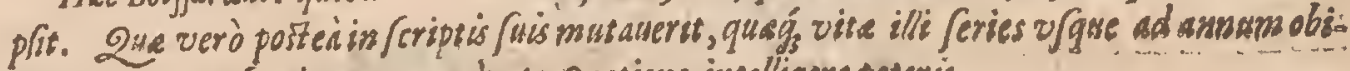
tus eivs 1609 fuerit, ex pracedente oratione intelligere poteris.

b E E 
14

\section{E P I C E D I A}

In obitum Clariffimi viri

\section{A R O L I C L V S I I.}

I.

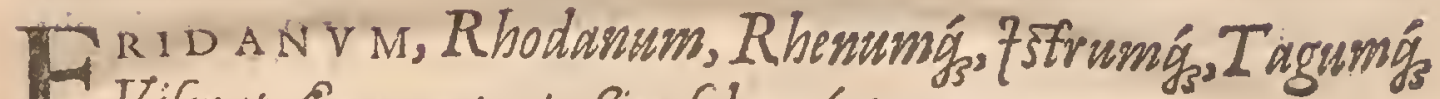

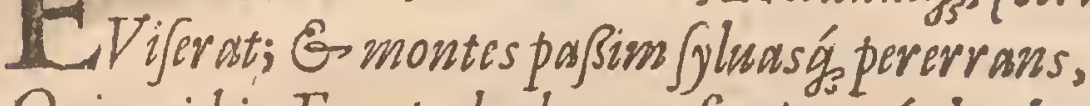

Qnicquid in Europa herbarum fruticumǵs latebat

Clufiada roirtus ftudio collegerat acri;

Tlle ctian quicquid tellus vel Americas, rol qua.

India furgentem ad Solem, coergénsuc cadentem

Innijum Europa $G$ nostris produxerat oris

(Qua voariis alli fcripferunt fingula longuis)

Doniarat Latio felicis munere penna,

Eximiis fulgens per totum lawdibus orberm.

At nuncè terris in calica templa veceptus

Aspectupropiore Deife Clujfus extlett.

Miriffcas nutu qui roires indidit herbis.

\section{I.}

T V L T V M bodie cuiquam fires herbaria debet;

N1. Debet Matihiole, Gdocte Lacunatibi.

Partribus hocipso debetur nomine Belgis

Gloria, non rollo deperituradie;

Clyfiade, Dodonao, doctoós Lobello,

Certis perpetturm rovinere nominibus.

Mattbioli, Vencti; Salamantica, frritta Lacune

Aterna ornanit laudis honore, typis.

At Dodonai, E Clusí, friptissys Lobelli

P'lantini furmmum praladedere decus.

Cefferunt fatis ommes, Te Parca, Lobelle,

Laudi quâ frueris din Jupereffe frsat.

Bon. Vvicanivs.

EIVSDEM 
EIVSDEM BON. VVLCANII

IN CLARISS. VIRI

EVERARDI VORSTII

\title{
Orationem funebrem
}

\section{IN OBITVM CAROLI CLVSI}

\author{
EPIGRA M M
}

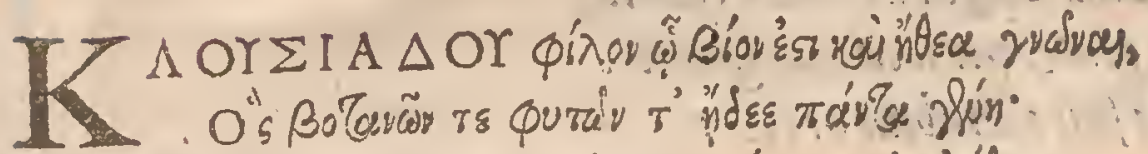

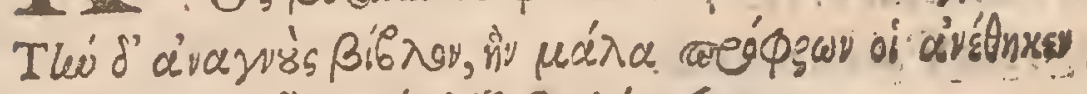

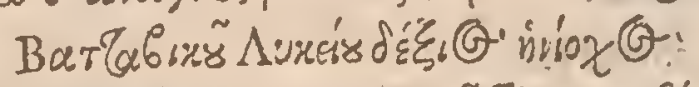

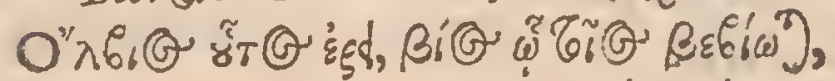

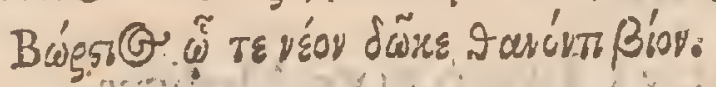

\section{Idem Latinè.}

CI V SIA D E fudia ES mores qui noffe laboras,

Herbacui nota ES plania propè omnis erati.

Perlegequas Bataui Rector Dooctorǵs. Lycei

Ills Jacrauit Vorstius inferias.

Felicem dices, talis cui vita pericta est,

(uigigu viro à calivita fecunda datur.'

intis

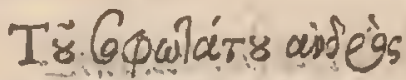

\section{KAPO $\Lambda$ OY K KOY \\ EIIIA $\Phi$ IN.}

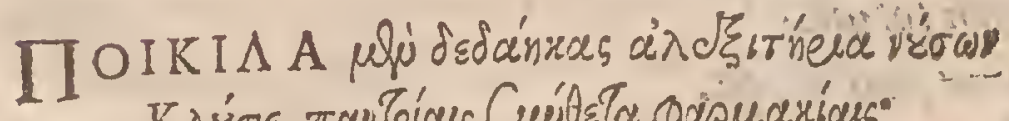

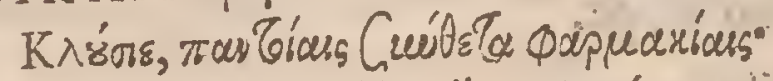

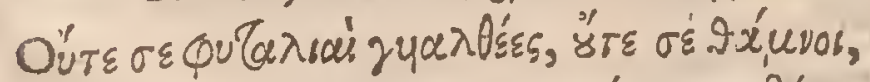

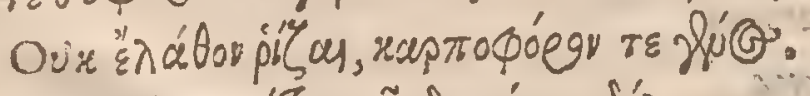

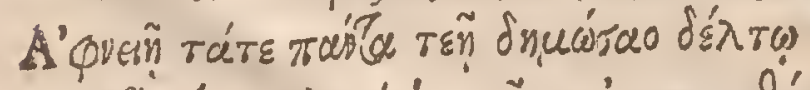

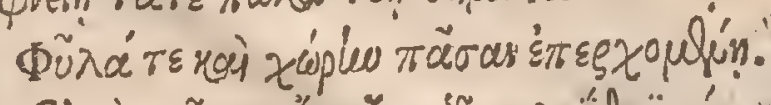

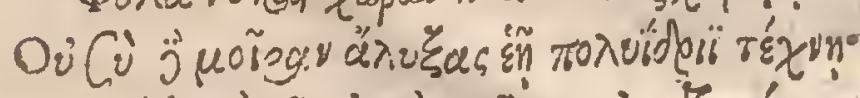

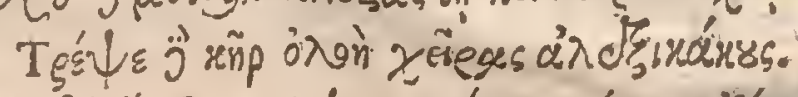

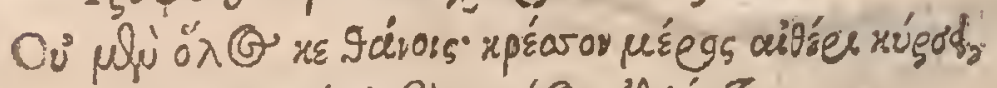

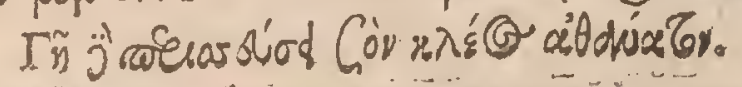


$x$

C A R OL I C L V S I I

\section{MTego Epitaphizm.}

To т A tibifuerant, Che $l$, pellentia morbos

1 Pharmacd diverfisconjocitis inodis.

Sed neque radices, nec te lat nexe Salubxes

Herbe, non frutices, fructiferum $q_{3}$ o enus;

Omnia que quondam oulgar as disite charia.

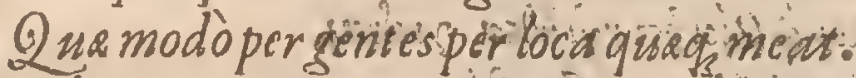

At tibi mors nullâ fuit evitabilis arte

Sedmedicas vicit tetricipariasianus.

Haud totus moriere tamen : pars maxima calo

$V$ inet, $\sigma$ in terris fama euperstes erit.

FEDERIC VS İA MOTIVS Medicus.

\section{In reliquias

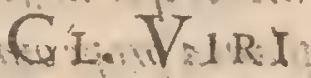 \\ CAROLI C L V I I

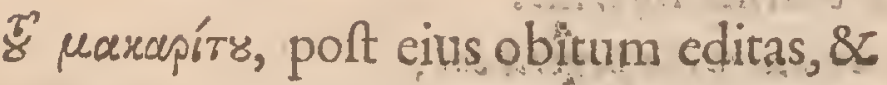 \\ ORATIONEM IT NNE BREM à $\mathrm{Cl}$. Viro
ELIO EVERARDO VORSTIO de eodem habitam.

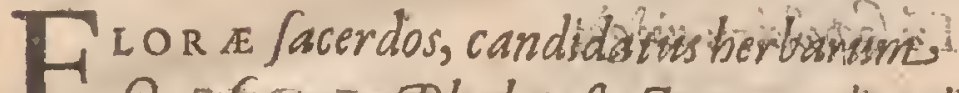

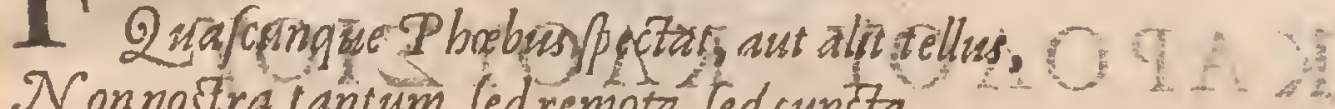

Connostrai anium, jedremota, fed cuncta,

2uam Pontris ambit, aut Oynphis in

Erepius orbi Clus Ius, tamen totum

Spargit per orbern non minus ups Hloress.

Fllum disertavoce Vorstius nuper,

Florum peritus EF perims berbarsm

Vita perenni confecrauit ES fama,

$V$ ict urus ipfe non minus celebrato.

Mufe corollam nectite boc ES boc digham

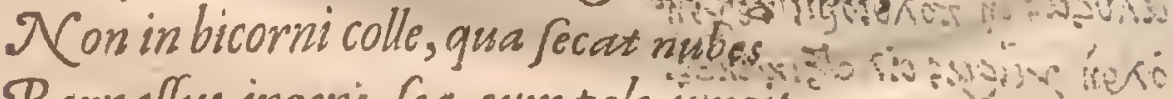

Parnafjus ingens, feq cumpolo iungit Quos preferatis Clufins dabit flores. 


\section{E P I T A P I V M diuini fenis \\ C.A ROLI C L V SII viri clariflmi, \& rei herbarix prin cipis.}

\section{$S$ C A Z O N.}

- TOS PEŚ, fepulchrum quilquis aspicis nostrum.,

1- Hic axiternum crede ver in hâc vornâ

Croco rubente, flo/culisǵg firare.

Ego ille faxo condor hoc, inexpletâ

Q ni mente dud'sw/cijcitabar, in campis

Q) u. procreatrix vis inerret, ojmagnum

Híc corpus animet : gue libedo pertente

Terram, ferenânocte quim leuic colum

Humorerorat, aut Fationitus lat as

Florum papillas purpurantium fufflat.

$\lambda$ Confrudia vorgime, nec asperte curis

$T$ ensête : ron mane togula fudatrix

Per mquict as duititum for:s egit : ,

Sed, dum ferè omnes diligent er bic errant,

Egointer berbas fulus, è clomo Ditis.

Sircis cabillis innocentiam prifci

-Ruris reduxt. fic mibi dies grati

Fulsé-ri: Jic plus octies decom maffe

Et tresperegi: quum nec interim tußis,

OMaliusve languor, nec grauedo me putris

Q.2ualjauit: oculos v/que dum meostandem

Dics iupremus, vis ǵ /auror fati

Gransure fommo preßst, \& femel longam

Downive noctem in 3 it, beunimis longam.

Lex aita vulu est fixa : quam licet dudum

Fingife iam viderer, baut tamen fugi.

Fcramus, bospes, hac acerba. debeniur

Diaioraworticorpora.illa, quam ftare

Suâ pustamus mole, iam labat terra

Nulátqui. Nata quse fuêre non mirum est

Perire; pert boc, ecce, quod fuit femper.

Scripli, PETRVS CVNAEVS。 
Nobilis ac prastantißimi roiri

\section{A R O L C L VSII.}

Q

VAM QVA M citatus pennipeság festines,

Hosbes, refiste./uadeo recufantem.

Uetist a prafens pompa te retardârit,

Q $u a$ Denecales Feria celebrantur.

Cernis cupreffos, or choragium luctius,

Hominesá, tristes mortualibus fumptis,

TMadidis $q_{3}$ ocellis. cernis in epulchreto,

Hîc quâ Lycaum perfonat Batausorum

Ip a as Aibenas prouocans Evechtheas,

FI anes ciere, lachrymisǵ, perfu/um,

Pullấá, veste, crinibusá dimißis,

Taxoǵ opertum Vorstium parentare;

Nonfulminato cuspiam è bidentali,

Scurrére, (ne nos Jicputes ineptive:)

Inuerfa fax bec, baǵ, imagines picta,

Et Jigna lecto que feruntur Orcino

Meliora pondent : nempe funus illustre,

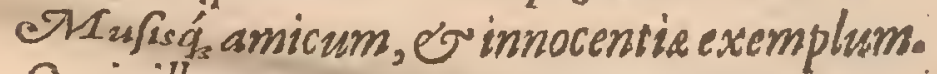

Q2 uis ille, queris, fit cuss Sacram fiat?

Cui ille planctus pectorum of lacertor mm:

Flle, ille magnus Clufus, pater Floras

Ceniusǵ, florsm, firpiumǵ, nutritor,

Post explicata Exotici Orbis $6 \mathrm{~N}$ Nostri

Diwer a ditis dona rara $N$ ature,

ISto quiefit conditorio. dixi.

At, ô Viator, paucula beunimis dixi.

Plura ip Se dicet, funerisǵ, Lasdator.

Addamus illud nos tamen, Virum salem.

Fuiffe, qualem fecla vix ferent vlla.

a vide pli.

niumlib.15.

lib.17.cap.1.

b Nempe

LAcrver.

N.I, Valerix

gêtis cogno-

men: Vide

lib.19.cap. 4

Apud Diod.

sicul. es A A flore poffes nominarevel Florum.

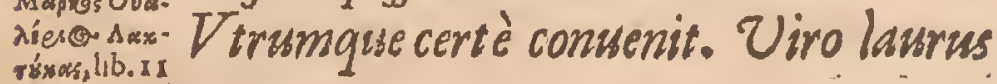


Condigna merces; dignus est ǵ florere Eternim; EG ora per volare doctorim. Nonille Candex, aut inutilis Truncus,

Stolo, Frutéxve. at Arbor admodium Felix.

Industria acris, diligentie rara;

Dumperuagatur rura $P$ anrionum quondam,

Plagasq́glisstrat tramites $q_{3}$ inaccefjos, cocas ǵvalles non veretur intrare: Britannias $q_{3}$ Hispaniasǵ percurrens Incognitorssm nos docet genus florum, Plantasá, of berbas prodit antè neglectas. Hac cura tant um Clus 10 fuit cordi; Fugiens docere iura pro tribunali, Que iusenis alme conferratus Astras Ev Vipiano didicit ES Modestino: Non ille auarus cuipiam dolos fruxit; (Haud nírat ollis inuidere the (auris,) Non fonorevfus tristium Kalendarum. Non prapotentum portulas falutator Captausit vdus, estuánsque, pallenság: Et ara centum, congiarium la $\beta_{i}$ Anteambulonis, iufit ille plorare; Fprosq́a reges, Eg greges togatorum. Non impudicis vifus est adhareres Submoenianas nec fecutus vxores: Calebs, fed instar Calitis fuit calebs. Reliquas procacis infolentias fecli Perofus, aureplaufúm que contemptor, Securres inter flof culos oberrabat; Ab inquietiquestibus proculvulgi, Questu volucrum per vireta contentus. Hinc pax Jepulto. Scara nec quiefcentem Strix olla turbat. nec cadauer allatrants Canes Iberi, Schoppinisve vespillo.

INNOCENTISSIM E ANIM E, AC MERENTISSIMIS MANIBVS

$$
\begin{aligned}
& \text { Petrvs SCrrverivs M. } \\
& \text { Epitaphium. }
\end{aligned}
$$


CLARISSIMI VIRI

\section{CAROLI CLVSII}

\section{A T R E B A T IS.}

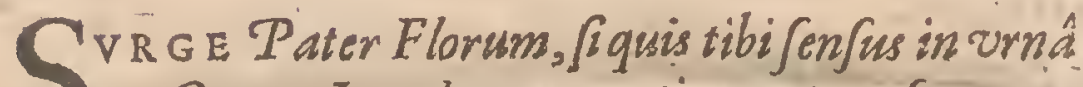

$\checkmark$ Qua te Lugdunum continet, atgue fouet

Magne fenex CL V S , quimaior A P O L LINE quosis

Ingenio es; ceptis addito Jumma tuis;

Nam nifiprima tui pars est exactat laboris,

Quam decorai lauro plebsóg opbúsque wo:

Orbibus ex mot is toto nostro orbe Bataus

Materis Oceanum/ug gerit Indipeta;

Quios ego! nicharum patris caput effera Morta

Fortior omniberbâ contumulaffet bumo:

Nil torrens terrere ignis, nil tristia Ponti

Flustra, ¿ Hyperborea vis violentaniuis:

$P$ lurima lisstrato retulifem munera misndo

Indeclinati maxuma pars operis:

Clase etenimnemus boc $N$ atura vo A PE R T A recludis,

$V t$ moto pateat cardine porta fatis.

T. dece fubfident mundi miracula montes

Tesquáque, quas es nullấ funt adeùnda viâ.

Saltibus ES fefe quecungue receßibus abdamts

$T$ elluxis cen us divite delicia;

Concedis nostris etiam reperirier hortis

Pannoniam, at que frndos, Spanica regna fimul.

Lugduni locus est fuperans vel Adonidis, iste

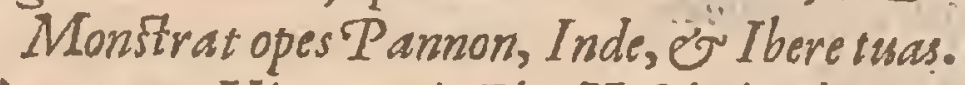

Hunc meus Hippocratis Flos, Ve all ǵs.Medullis

PAVIVs aritauit dispoj of â Serie,

Magnificum cimelium opsm, admirabilis arte

$\mathcal{X}$ Costrâ; of qui $C_{L}$ v s I cludere dignus opies.

Dispercam, Hippocrates ! s quifquarn dignior alier

Tangere Thoboo tanta Viretapede.

Verumignofe Senex mulio guod membra labore

Lafla fat is, placidum nactáque morte locum 'Ad folitos ressocem ingratus, deménsque labores;

Nam mea mens peccat turbida, morte tuá.

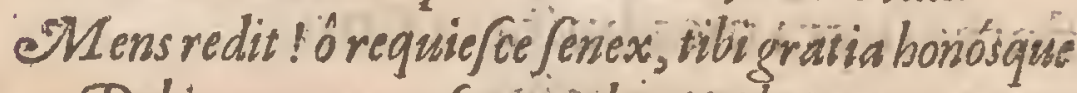

Debita, perpetuafurget ab ori tisbe; 
Surgere te C Lv s I nec opuis, namparite fuperstes.

Es potiore tusi; $\mathrm{P}$ avivs evigilat.

Decidusum Sequitur for en fubmit]us ểंdem

Stirpe, quod hasd certas commonet effe rices.

Cafaribus charus vernanti fore inuent $a_{\text {, }}$

Et fructus fenii gestiit ippes Scbola.

Absipfo Senio fimmpfit twa gloria rires,

Gloria qua nullá est interitura die.

Fatalis lustris eadem labentibus orbi,

Atque operi. Manes vinite Clufficio

\section{XPONOTPAФIKON.}

BIs qVInqVeoCtenos, qVatVorqVe peregerat annos

CLVsIVs Vt Menfls LabItVr, ad fVperos

pertaPVs terras penetraVIt CLVfIVs hortos,

LVX VbI perpetVo Vere trahenda potens:

Angerius T.F.Clutius. 


\section{Viro nominatiffimo, \\ C A R OLO C L V S O,}

aui noftri Principi.Botanico.

₹ Ita Tollius

Famam n10. minat Epit.
ad Attic.

V L T I M A mystacio ex Medico quierenda, Beate

Gloria, defunctor animâ eternißime C I v Sì,

$\mathcal{N}$ f fatum, * aternámque piorum carmina vatum.

Tot collaudafjent vitam, calóque locafjent:

Quiscum triste Apium, geminam Abrotanúmque, T bymiumgise,

Omnigenisque berbeftruices meroris alumnas,

Florésque, umbellásque, comas, frondésque, corymbósque,

Et pullas capital fugiens Dea prafica Laurus

Infer lauriuoris plenácum adpergine pompis.

Plini vmbre beu! diui maiores, entlie diui

Suada Theophrasti, atque Anazarbeà alta Tedaci

Diusa Diofcoridis! quos nunc exofa Beati

Pars melior tradux floret calestibus bortis:

Florâ nate, pater florum, berbarúm que, cui ipfa

Squallentis feriles enixo in luminis auras

Tellus floridulis pregnantem enitier aruis,

Aique aperive oculos, queîs tot mortalibus annos

* Beasi no. mipis veri-

loquium,

hunc iam

natura iaguts

meesas Évas

Telíus opes,

herbas, frus-

cices, Gotes.

coocluferat,

quò \& ocu.

locū extyonon.

deri poteft;

enien dero.

trinati.

† Liecat re-

nià Perfarum

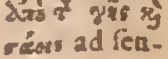

Sumbü pro

Gracis tsa -

daci.

* Cluserat ingenuos fructus, florumve profundas

Aurragine iamte beu! delato ad confcia terre

Templa, onde adjurgurs florum prolimina, $t g a$ al

Inuidere fesas fuperis; radicibus ipsâ

Tellure occlufus cacas protrudere vitas,

Fruitumque animare lutum, of variare colorum

Tot vultus, peciésgue, ant helásque admirandas!

Fmò ip fe in varios flores mut arier, ip/us

Flos florum, Florequ, EO qui huic operant tur Alumni

Flores, indictásque berbas, exotica calo

Nostroignota orbi, Bataua o miracula genti

Ingentique orbi, propriis qui floribus orbis

Iam facit indigeti, totus quem floridus orbis

Terrarímque Dii fratres, nymphég forores,

Herbarum florúmque Animam, Geniumque Iousémqse,

Naturéque funm mysten, fratrémque magistrum

Lacteolo indigitem per calos tramite ducant.

L. M. Q.

Coronidem adpofui $H$. DEIMA NHORSTIVS xre rళे pargeítr CIVSII non Comico, at Botanico obxratus. 


\section{A NVS RVTGERSIVS}

\section{Euerardo Vortio V.C.}

G IC tibiconcedat medicanti Pergamiss arte,

$\omega$ Neccertet dextradextera Coa tue;

Sic coëant queifcumque facras admoueris herbas

Uulnera, nec dubiam fentiat eger opem;

Seuágune crwdelis cefent cont agia morbi,

Et fugiant esccis victa venena tusis

$U t$ mibi le non antè oculis tamgrata legendam:

Obtuslit eternácharta notata manu,

Crudeles obitus que raptimoret amici,

Et iacit ad vidusos tristiaverba rogos,

Q usalia Jub densâ ramorum concinit umbrâ

Dum gemit abfumptum Dauliamater Ityn,

Qualia cantabat Geticis in rupibus Orpheus

Euridyces questus alterafata fus.

Hec quoque venturi difcant te vate nepotes,

Exequias aurm posteritatis eat.

Quid renuis frustra? non est tua pagina, $\mathrm{VOR} \overline{\mathrm{STI}}$,

Q ue lacrymas fere posteritatis babet.

\section{E I V S D E M \\ De Civsir obitu.}

TIDERAT astrorum motus, caus asque notarat:

Emenfus ftellas, of totum Clus lus orbem,

In gernino quantsum cardine Phobus obit,

Iam didics coelum, quid adbuc nifis terra relicta est?

Hine quoque, (ed maior, fama petetur, ait.

Nec mora, Telluris facras accingitur berbas,

Et gremio quicquid omniparente foust.

Fam penetrat Gangen, videt hunc iam Medus Hydaspes,

Et qui Septeno gurgite Nile fluis.

Omnias quarebat. querenti defuit orbis,

Ulterius nec quo progrederetur erat.

Nunc iacet bic. Tellus varias superaddidit berbas,

Et tumulo lacrimis bumida ferta dedit.

Qui coluit flores, ES facre femina matris,

Credibile est illum fic voluif]e tegi. 


\section{A L I V D,}

In tumulum GLvSII.

PVOD iacet boc tumulo fivis nouife, viator, Exunias Clusi continet istelocus, Con, tectes fenio, fractas morbisidie maltique, Sunimáque rix quarim frinxerat offacutis. Sed tameningeniwrn, fedmens divinior butis Hess maie formati corporis hospes erat. Kon querimur raptum. Q 2uidquid mors inuida dempjit, Frigenio nobis reddidit ille Jwo.

\section{A L I V D.}

C v Clusí corpus tumulo Natura locaret,

Quem marens manibus fecerat ip a f uis, Ingerrunt, tollénsque manus ad fidera, T erre Hocpeperi, calum cetera, dixit, babet.

\section{A . L I V. D.}

Q vi videt bos flores tumuli de vertice nalci, O Hec cineritellus vlt ima dona dedit. O bene, quad iumuloclandatur Clusius isto! Quicolust flores, foribus ille iacet.

\section{A L I V D,}

\section{ad Cardi Clusis Manes.}

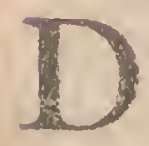

IVINI manes, Ef tantinominis oumbre,

$E_{\iota}$ Clusî quicquid hor tegit orna rnsis Accipite exigurum manjuripignus arionis,

Dum liceat nobis condere mäus opius. Accipite, er quamus parium ne pernite munus. Hac pietas femper digna fanorefuit. 